\title{
Effect of back massage on relieving pain during labour: a systemic review
}

\author{
Suman Choudhary ${ }^{1}$, Prasuna Jelly², Prakash Mahala ${ }^{3 *}$, Amali Mery ${ }^{2}$
}

\begin{abstract}
${ }^{1}$ Department of Infection Control, ${ }^{2}$ College of Nursing, ${ }^{3}$ Department of Emergency Medicine, All India Institute of Medical Sciences, Rishikesh, Uttarakhand, India
\end{abstract}

Received: 23 March 2021

Accepted: 27 April 2021

\author{
*Correspondence: \\ Prakash Mahala, \\ E-mail: prakashjpmmahala@gmail.com
}

Copyright: (c) the author(s), publisher and licensee Medip Academy. This is an open-access article distributed under the terms of the Creative Commons Attribution Non-Commercial License, which permits unrestricted non-commercial use, distribution, and reproduction in any medium, provided the original work is properly cited.

\begin{abstract}
Managing labour pain is a challenging concern for nurses who involved in care of mothers during labour and child birth. Massage is a primordial method that has been generally employed during labour, however, relatively little study has been assumed examining the effects of massage on women during labour. The study insistent pain allied with labour may negatively impact mother further transforming to foetus, frequently varying the childbirth course. The techniques of health care humanisation mention that women in labour should have the chance to relieve their pain with pharmacological and non-pharmacological methods. The systemic review examines literature on effectiveness of back massage to relieve labour pain from 2013 to 2019. The electronic database reviewed for the systemic review included PubMed, Medline, nursing health journal, Google scholar etc by including relevant key words. Ten studies were included in the systemic review. The available literature on non-pharmacological method provides evidence as a back massage is effective to reduce labour pain. The aim of the present review is to examine the effect of back massage as a method to relieve labour pain and give comfort to mother. The experience of labour pain is different in women and it is affected by several psychological and physiological factors and its intensity may vary significantly. During labour majority of women need pain relief. Strategies of pain management include pharmacological and nonpharmacological intervention. Evidence suggests that non pharmacological methods are helpful to reduce labour pain. We identified 10 reviews out of 110 for inclusion within this review. All studies on back massages show that it is effective to relieve pain during labour.
\end{abstract}

Keywords: Back Massage, Labour, Pain, Effect, Relieving

\section{INTRODUCTION}

The pain of labour is considered to be the most severe pain that a woman always has to face. Labour pain can be intense with fear; body tension and anxiety make it worse. Many techniques are used by women's in labour such as, applying warmth or cold, massaging touching, bathing, grooming, hypnosis, music and emotional support also such as continuous reassurance, encouragement, protective guidance, information facility and non-medical advice. ${ }^{1}$ A study showed that labour support measures such as back massage, deep breathing exercise, and positioning were effective in reducing the labour pain. Because during labour, every woman experiences some degree of pain, but perception of pain is different from one individual to another. ${ }^{2}$ World-wide, every day around 800 women die due to preventable causes related to pregnancy and childbirth, 20\% women are belonging from India. Due to preventable pregnancy related death of women's in India is estimated 44,000. Childbirth is a certain time and one of the largest events for the emotional life for a pregnant woman. Pregnant are worried about the pain during labour and concern how to deal with pain. Most important cause of denying natural vaginal delivery is fear of delivery pain. So rates of caesarean sections is increasing due to short awareness of 
the different ways to reduce labour and it is also issue. ${ }^{3}$ The purpose of this systemic review was assessing the effectiveness of non-pharmacological method to relieve pain in labour and helping in reducing caesarean sections and increase the tendency of mothers to natural vaginal delivery in hospitals of our country. Massage contains influencing soft tissues of body. It is used to help relax muscles tense and to help calm. There are different massage techniques may help to benefit to reduce pain during labour such as lower back massage, smooth strokes, also called effleurage, counter pressure and hip squeezes. $^{4}$ Massage stimulates our body to release endorphins, the natural pain-killing, mood-lifting chemicals produced in the brain. In the first stage of labour massage may reduce anxiety and ease pain. It also helps to cope with contractions by making feel less intense and more manageable. ${ }^{5}$

\section{Aims and objectives}

The overall aim of current investigation is to review the literature that identifies the effectiveness of back massage on labour pain relief.

\section{METHODS}

Articles from electronic sources and other sources were reviewed intensely related to the topic. This review intends to find out the studies which shown the effect of back massage in experimental and control group in different health care setting. The electronic databases reviewed for the systemic review of the literature are: PubMed, Medline, nursing health journal, Google scholar and the selected articles were from the relevant journals of health care sciences. MESH search parameters were included to find out the studies which were publishes during the years 2013 to 2019, which has access to full articles content, meeting the inclusion and exclusion criteria and the studies shown effectiveness of back massage on labour pain.

\section{Analysis and data extraction}

Inclusion criteria for literature search included of the quasi experimental quantitative studies in health care centre and literature related to women who received back massage during labour to relieve labour pain were included for reviews which are written in English language and the studies of back massage on mothers with other medical conditions were excluded. A total of 110 studies related to back massage in reducing pain during labour were originated in the search at initial stage. 46 studies were excluded due to non-availability of full text and 46 research studies were dropped out from the present review based on inclusion and exclusion criteria. Finally, 10 studies were included in the systemic review making sure that each of the selected studies outcome measurement related to the effect of back massage on labour pain (Figure1).

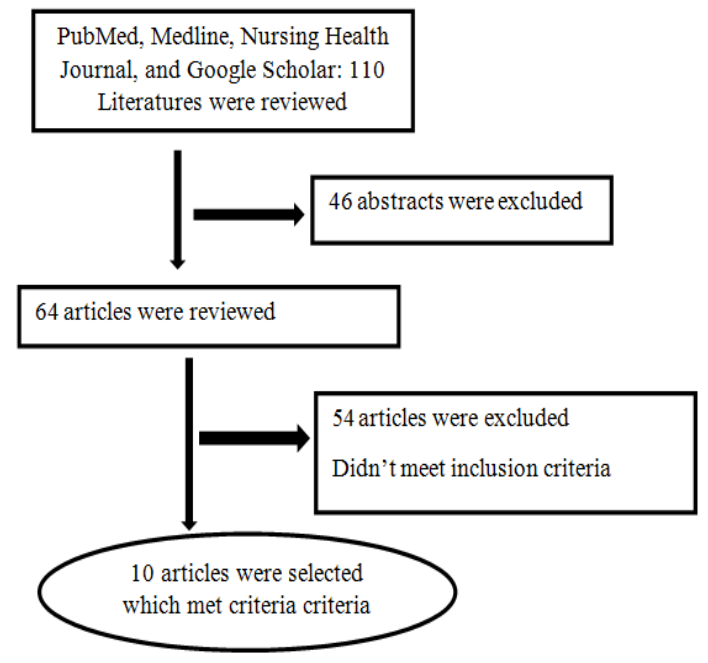

Figure 1: Flow chart of literature selection.

All the members of review screened the topic and reviews, based on inclusion criteria separately. During study if there was conflict on certain topic, it was resolved by mutual understanding and reviewing further studies. Data extraction and maintenance of accuracy was done including author, year, country in which study has been done setting, design, sample, tools used in study, data analysis and result (Table1).

\section{Quality appraisal criteria}

Standardized visual Analogue Scale used as instrument was utilized to assess the labour pain. This included tools for pre-test and post-test control group and case control Studies. This standardized instrument is supporting this study as the result reviews are valid, results are statistically significant, $(\mathrm{p}<0.05)$ and literature shows that massage is effective in reduction of labour pain.

\section{Data synthesis}

Synthesis demonstrates analysis of papers used for literature review. Synthesis is done by two authors but each of them synthesizes it separately. Data collected and summarized based on relevant studies. Research data was organised in such a manner that will help to find-out certain questions related to reduction of pain during labour. There are varieties of methods used to decrease pain during labour. These reviews help to formulate a guideline to provide benefit to patient during the procedure of massage for reduction of pain during labour.

\section{RESULTS}

Based on the inclusion criteria, 10 articles published were closely relevant were included in the present review. Details of the studies are given below; total 10 research studies which used Quasi experimental research design were included. 
Table 1: Characteristics of the study has been reviewed.

\begin{tabular}{|c|c|c|c|c|c|c|c|}
\hline $\begin{array}{l}\text { Ref. } \\
\text { no. }\end{array}$ & Aim & Method & $\begin{array}{l}\text { Sample size, } \\
\text { setting }\end{array}$ & Measurement & $\begin{array}{l}\text { Sampling } \\
\text { technique }\end{array}$ & $\begin{array}{l}\text { Data } \\
\text { analysis }\end{array}$ & Result \\
\hline 2 & $\begin{array}{l}\text { Effectiveness } \\
\text { of labour } \\
\text { support } \\
\text { measures on } \\
\text { pain } \\
\text { perception of } \\
\text { mothers in } \\
\text { labour }\end{array}$ & $\begin{array}{l}\text { quasi- } \\
\text { experimental } \\
\text { (multiple } \\
\text { time series } \\
\text { design) }\end{array}$ & $\begin{array}{l}\text { Primi } \\
\text { mothers, } \\
\text { (sample size } \\
60,30 \\
\text { experimental } \\
\text { and } 30 \\
\text { control } \\
\text { group) }\end{array}$ & $\begin{array}{l}\text { Numerical } \\
\text { pain intensity } \\
\text { scale }\end{array}$ & $\begin{array}{l}\text { non- } \\
\text { probability } \\
\text { consecutive } \\
\text { sampling } \\
\text { technique }\end{array}$ & ANOVA & $\begin{array}{l}\text { There was a } \\
\text { significant } \\
\text { difference in } \\
\text { experimental and } \\
\text { control groups. } \\
\mathrm{F}=3.931 \text { is more } \\
\text { than } \mathrm{F}(1,58) \\
\mathrm{p}<0.05=1.534 \text { to } \\
\text { table value } \\
\text { which shows } \\
\text { that it was } \\
\text { statistically } \\
\text { significant at } \\
0.05 \text { levels }\end{array}$ \\
\hline 6 & $\begin{array}{l}\text { To determine } \\
\text { the } \\
\text { effectiveness } \\
\text { of paced } \\
\text { breathing and } \\
\text { back massage } \\
\text { in labor pain } \\
\text { Management. }\end{array}$ & $\begin{array}{l}\text { Quasi } \\
\text { experimental } \\
\text { design }\end{array}$ & $\begin{array}{l}\text { Antenatal } \\
\text { mothers } \\
\text { (sample size } \\
200,100 \\
\text { experimental } \\
\text { and } 100 \\
\text { control } \\
\text { group) } \\
\text { Orotta } \\
\text { National } \\
\text { referral } \\
\text { maternity } \\
\text { hospital }\end{array}$ & $\begin{array}{l}\text { Visual } \\
\text { analogue } \\
\text { scale }\end{array}$ & $\begin{array}{l}\text { Purposive } \\
\text { sampling }\end{array}$ & $\begin{array}{l}\text { Mean SD } \\
\text { Paired ' } t \text { ' } \\
\text { used }\end{array}$ & $\begin{array}{l}\text { There was } \\
\text { significant } \\
\text { difference } \\
\text { between pre } \\
\text { assessment and } \\
\text { post assessment } \\
\text { level of pain } \\
\text { (value }=12.961 \text { ) } \\
\text { in group II } \\
\mathrm{p}=0.5 \text {. }\end{array}$ \\
\hline 7 & $\begin{array}{l}\text { Effect of } \\
\text { massage } \\
\text { treatment } \\
\text { using } \\
\text { frangipani } \\
\text { aromatherapy } \\
\text { oil to reduce } \\
\text { the childbirth } \\
\text { pain } \\
\text { intensity. }\end{array}$ & $\begin{array}{l}\text { Quasi } \\
\text { experimental } \\
\text { research } \\
\text { design } \\
\text { (case-control } \\
\text { study) }\end{array}$ & $\begin{array}{l}\text { Pregnant } \\
\text { women } \\
\text { (sample size } \\
70,35 \\
\text { experimental } \\
\text { and } 35 \\
\text { control) } \\
\text { Pembantu } \\
\text { Dauh Puri } \\
\text { health center } \\
\text { Denpasar, } \\
\text { Bali, } \\
\text { Indonesia }\end{array}$ & $\begin{array}{l}\text { Numerical } \\
\text { pain rating } \\
\text { scale }\end{array}$ & $\begin{array}{l}\text { Non } \\
\text { probability } \\
\text { consecutive } \\
\text { sampling }\end{array}$ & $\begin{array}{l}\text { Kolmogorov- } \\
\text { Smirnov } \\
\text { test, Mann } \\
\text { Whitney test } \\
\text { at } 95 \% \text { CI } \\
\text { using SPSS } \\
\text { version } 19 .\end{array}$ & $\begin{array}{l}\text { Significant } \\
\text { difference } \\
(\mathrm{p}<0.01) \text { in the } \\
\text { response was } \\
\text { observed after } \\
\text { the massage } \\
\text { therapy, with the } \\
\text { decrease of } \\
\text { severe pain in } \\
\text { respondents in } \\
\text { the treatment } \\
\text { group. }\end{array}$ \\
\hline 8 & $\begin{array}{l}\text { Effects of } \\
\text { massage } \\
\text { during labor } \\
\text { on pain and } \\
\text { length of } \\
\text { delivery in } \\
\text { nulliparous } \\
\text { women }\end{array}$ & $\begin{array}{l}\text { Quasi- } \\
\text { experimental } \\
\text { study (case- } \\
\text { control } \\
\text { study) }\end{array}$ & $\begin{array}{l}\text { Nulliparous } \\
\text { women } \\
\text { sample size } \\
100(50 \\
\text { experimental } \\
\text { and } 40 \\
\text { control } \\
\text { group) } \\
\text { social } \\
\text { security } \\
\text { hospital of } \\
\text { Ardabil, } \\
\text { Iraq, }\end{array}$ & $\begin{array}{l}\text { Numerical } \\
\text { pain rating } \\
\text { scale }\end{array}$ & $\begin{array}{l}\text { non- } \\
\text { probability } \\
\text { purposive } \\
\text { sampling } \\
\text { method }\end{array}$ & $\begin{array}{l}\text { Chi-Square, } \\
\text { t-test and } \\
\text { Mann- } \\
\text { Whitney tests }\end{array}$ & $\begin{array}{l}\text { There was } \\
\text { Statistically } \\
\text { highly } \\
\text { significant at } \\
\mathrm{P}<0.05 \\
\text { Massage reduced } \\
\text { the intensity of } \\
\text { labor pain } \\
\text { (p<0.001) and } \\
\text { length of the first } \\
\text { stage of labor } \\
(\mathrm{p}<0.001)\end{array}$ \\
\hline
\end{tabular}

Continued. 


\begin{tabular}{|c|c|c|c|c|c|c|c|}
\hline $\begin{array}{l}\text { Ref. } \\
\text { no. }\end{array}$ & $\operatorname{Aim}$ & Method & $\begin{array}{l}\text { Sample size, } \\
\text { setting }\end{array}$ & Measurement & $\begin{array}{l}\text { Sampling } \\
\text { technique }\end{array}$ & $\begin{array}{l}\text { Data } \\
\text { analysis }\end{array}$ & Result \\
\hline 9 & $\begin{array}{l}\text { Effect of } \\
\text { effleurage on } \\
\text { Pain Severity } \\
\text { and Duration } \\
\text { of labor among } \\
\text { laboring } \\
\text { Women. }\end{array}$ & $\begin{array}{l}\text { Quasi } \\
\text { experimental } \\
\text { (case-control } \\
\text { study) }\end{array}$ & $\begin{array}{l}\text { Pregnant } \\
\text { women } \\
\text { sample size } 60 \\
\text { (30 } \\
\text { experimental } \\
\text { and } 30 \\
\text { control) } \\
\text { Ismailia } \\
\text { university } \\
\text { hospital }\end{array}$ & $\begin{array}{l}\text { Maternal } \\
\text { interviewing } \\
\text { schedule, } \\
\text { visual } \\
\text { analogues } \\
\text { scale (VAS) }\end{array}$ & $\begin{array}{l}\text { Non } \\
\text { probability } \\
\text { purposive } \\
\text { sampling } \\
\text { method. }\end{array}$ & $\begin{array}{l}\text { Chi } \\
\text { Square } \\
\text { test, t test } \\
\text { and } \\
\text { Mann } \\
\text { Whitney } \\
\text { test }\end{array}$ & $\begin{array}{l}\text { Statistically } \\
\text { highly } \\
\text { significant } \mathrm{p}<0.05 \text {. } \\
\text { The incidence of } \\
\text { lower score pain } \\
\text { section was } \\
\text { significantly } \\
\text { lower in the } \\
\text { study group } \\
\text { compared to } \\
\text { control group at } \\
\text { cervical } \\
\text { dilatations of } 6,8 \\
\text { and } 10 \mathrm{~cm} \text {. There } \\
\text { were significant } \\
\text { differences in } \\
\text { relation to pain } \\
\text { perceived } \\
\text { between two } \\
\text { groups. }\end{array}$ \\
\hline 10 & $\begin{array}{l}\text { Effectiveness } \\
\text { of abdominal } \\
\text { effleurage on } \\
\text { labor pain } \\
\text { intensity and } \\
\text { labour } \\
\text { outcomes } \\
\text { among } \\
\text { nullipara } \\
\text { mothers during } \\
\text { first stage of } \\
\text { labor }\end{array}$ & $\begin{array}{l}\text { Quasi- } \\
\text { experimental } \\
\text { study (Case- } \\
\text { control } \\
\text { study) }\end{array}$ & $\begin{array}{l}\text { Pregnant } \\
\text { women } \\
\text { sample size } 60 \\
\text { (30 } \\
\text { experimental } \\
\text { and } 30 \text { control } \\
\text { group) civil } \\
\text { hospital, } \\
\text { Ambala, } \\
\text { Haryana, } \\
\text { India. Civil } \\
\text { Hospital }\end{array}$ & $\begin{array}{l}\text { Numeric pain } \\
\text { intensity scale }\end{array}$ & $\begin{array}{l}\text { Non } \\
\text { probability } \\
\text { purposive } \\
\text { sampling } \\
\text { technique }\end{array}$ & $\begin{array}{l}\text { paired } \\
\text { "t } t " \text { test } \\
\text { ANOVA }\end{array}$ & $\begin{array}{l}\text { The computed } \\
\text { ' } \mathrm{t} \text { ' value was } \\
\text { found to be } \\
\text { statistically not } \\
\text { significant } \\
(\mathrm{t}=0.64, \mathrm{p}=0.52) \\
\text { at } 0.05 \text { level of } \\
\text { significance } \\
\text { which shows that } \\
\text { the difference in } \\
\text { pain intensity } \\
\text { score was not a } \\
\text { true difference. }\end{array}$ \\
\hline 11 & $\begin{array}{l}\text { Effectiveness } \\
\text { of routine back } \\
\text { massage versus } \\
\text { jasmine oil } \\
\text { back massage } \\
\text { versus coconut } \\
\text { oil back } \\
\text { massage on } \\
\text { labour pain } \\
\text { among } \\
\text { parturient } \\
\text { mothers. }\end{array}$ & $\begin{array}{l}\text { Quasi } \\
\text { experimental } \\
\text { (case-control } \\
\text { study) }\end{array}$ & $\begin{array}{l}60 \text { parturient } \\
\text { mothers who } \\
\text { were allotted } \\
\text { randomly in } \\
\text { three groups } \\
\text { each having } \\
20 \text { mothers, } \\
\text { tertiary } \\
\text { hospital, } \\
\text { Banglore. }\end{array}$ & $\begin{array}{l}\text { Numerical pain } \\
\text { scale and } \\
\text { visual } \\
\text { analogue scale }\end{array}$ & $\begin{array}{l}\text { Simple } \\
\text { random } \\
\text { sampling }\end{array}$ & $\begin{array}{l}\mathrm{t} \text { test and } \\
\text { ANOVA }\end{array}$ & $\begin{array}{l}\text { The mean post } \\
\text { interventional } \\
\text { score of coconut } \\
\text { oil back massage } \\
5.84 \text { is lower } \\
\text { than the mean } \\
\text { pre } \\
\text { interventional } \\
\text { score } 7.91 \text { in } \\
\text { which calculated } \\
\text { t value within } \\
\text { group was } 11.82 \\
\text { ( } p=0.001 \text { ) }\end{array}$ \\
\hline 12 & $\begin{array}{l}\text { Effectiveness } \\
\text { of olive oil } \\
\text { back massage } \\
\text { on reduction of } \\
\text { labor pain } \\
\text { during first } \\
\text { Stage of Labor }\end{array}$ & $\begin{array}{l}\text { Quasi- } \\
\text { experimental } \\
\text { (post-test- } \\
\text { only control } \\
\text { group } \\
\text { design) }\end{array}$ & $\begin{array}{l}60 \\
\text { primigravida } \\
\text { mothers } \\
\text { (30 control, } 30 \\
\text { experimental } \\
\text { group) } \\
\text { Jamia } \\
\text { Hamdard, } \\
\text { Delhi }\end{array}$ & $\begin{array}{l}\text { Numeric rating } \\
\text { pain scale }\end{array}$ & $\begin{array}{l}\text { Purposive } \\
\text { sampling } \\
\text { technique }\end{array}$ & $\mathrm{t}$ test & $\begin{array}{l}\text { Statistically highly } \\
\text { significant } \mathrm{p}<0.05 \text {. } \\
\text { Calculated ' } \mathrm{t} \text { ' } \\
(12.09) \text { was more } \\
\text { that the table } \\
\text { value, hence there } \\
\text { was a significant } \\
\text { difference in the } \\
\text { level of pain in the } \\
\text { experimental and } \\
\text { control group. }\end{array}$ \\
\hline
\end{tabular}




\begin{tabular}{|c|c|c|c|c|c|c|c|}
\hline $\begin{array}{l}\text { Ref. } \\
\text { no. }\end{array}$ & Aim & Method & $\begin{array}{l}\text { Sample size, } \\
\text { setting }\end{array}$ & Measurement & $\begin{array}{l}\text { Sampling } \\
\text { technique }\end{array}$ & $\begin{array}{l}\text { Data } \\
\text { analysis }\end{array}$ & Result \\
\hline 13 & $\begin{array}{l}\text { Effectiveness of } \\
\text { ice massage in } \\
\text { reduction of } \\
\text { labour among } \\
\text { primigravida } \\
\text { mothers }\end{array}$ & $\begin{array}{l}\text { Quasi } \\
\text { experimental } \\
\text { (case-control } \\
\text { study) }\end{array}$ & $\begin{array}{l}60 \\
\text { primigravida } \\
\text { mothers } \\
\text { (30 control } \\
\text { group, } 30 \\
\text { experimental } \\
\text { group) infant } \\
\text { Jesus hospital, } \\
\text { Madurai } \\
\text { District Tamil } \\
\text { Nadu }\end{array}$ & $\begin{array}{l}\text { Modified } \\
\text { visual } \\
\text { analogue } \\
\text { scale }\end{array}$ & $\begin{array}{l}\text { Purposive } \\
\text { sampling } \\
\text { technique }\end{array}$ & $\mathrm{t}$ test & $\begin{array}{l}\text { There was a } \\
\text { significant } \\
\text { difference in } \\
\text { experimental } \\
\text { and control } \\
\text { groups shows } \\
\text { that it was } \\
\text { statistically } \\
\text { significant at } \\
0.05 \text { levels }\end{array}$ \\
\hline 14 & $\begin{array}{l}\text { A study to } \\
\text { assess the } \\
\text { effectiveness of } \\
\text { back massage } \\
\text { on pain among } \\
\text { primigravida } \\
\text { mothers during } \\
\text { first stage of } \\
\text { labour }\end{array}$ & $\begin{array}{l}\text { True } \\
\text { experimental } \\
\text { study (pre-test } \\
\text {-post-test- } \\
\text { control group } \\
\text { design) }\end{array}$ & $\begin{array}{l}30 \\
\text { primigravida } \\
\text { mothers } \\
\text { (15 control } \\
\text { group, } 15 \\
\text { experimental } \\
\text { group) Govt. } \\
\text { maternity } \\
\text { hospital } \\
\text { Anantnag, } \\
\text { Kashmir }\end{array}$ & $\begin{array}{l}\text { Wonback's } \\
\text { pain } \\
\text { assessment } \\
\text { scale }\end{array}$ & $\begin{array}{l}\text { Random } \\
\text { sampling }\end{array}$ & $\mathrm{t}$ test & $\begin{array}{l}\text { There was a } \\
\text { significant } \\
\text { difference in } \\
\text { experimental } \\
\text { and control } \\
\text { groups shows } \\
\text { that it was } \\
\text { statistically } \\
\text { significant at } \\
0.05 \text { levels }\end{array}$ \\
\hline
\end{tabular}

Table 2: Major findings.

\begin{tabular}{|c|c|c|}
\hline Theme & Findings & Source \\
\hline \multirow{10}{*}{$\begin{array}{l}\text { Effectiveness of } \\
\text { back massage on } \\
\text { pain among } \\
\text { Primigravida } \\
\text { mothers during } \\
\text { first stage of labour }\end{array}$} & $\begin{array}{l}\text { There was a significant difference between the mean pain scores of } \\
\text { mothers in experimental group and control groups. }\end{array}$ & Choudhary et al 2018 \\
\hline & $\begin{array}{l}\text { There were significant differences in pre assessment level of labour } \\
\text { pain with that of the post assessment level of labour pain }\end{array}$ & Kavitha et al 2013 \\
\hline & $\begin{array}{l}\text { Significant difference }(\mathrm{p}<0.01) \text { in the response was observed after } \\
\text { the massage therapy, with the decrease of severe pain in respondents } \\
\text { in the treatment group }\end{array}$ & Sriasih et al 2019 \\
\hline & $\begin{array}{l}\text { There were significant differences in relation to pain perceived } \\
\text { between the two groups. }\end{array}$ & Khavandizadeh et al 2014 \\
\hline & $\begin{array}{l}\text { There was a significant difference between the mean pain scores of } \\
\text { mothers in experimental group and control groups }\end{array}$ & Zaghloul et al 2018 \\
\hline & $\begin{array}{l}\text { There is no significant difference in pain intensity score of mothers } \\
\text { in experimental group and control groups }\end{array}$ & Rashmi et al 2013 \\
\hline & $\begin{array}{l}\text { There is significant difference between pre and post interventional } \\
\text { pain score. There is no statistical significant association was found in } \\
\text { post-assessment level of labour pain perception of experimental and } \\
\text { control groups }\end{array}$ & Devi et al 2016 \\
\hline & $\begin{array}{l}\text { There is statistically significant difference between the mean pain } \\
\text { scores of mothers in experimental group and control groups, there } \\
\text { was significant difference in the level of pain in the experimental } \\
\text { group before and after administration of olive oil massage. }\end{array}$ & Chauhan K et al 2016 \\
\hline & $\begin{array}{l}\text { A significant difference at the level of } \mathrm{p}<0.05 \text { was found between } \\
\text { the experimental and control group on labour pain, there was } \\
\text { significant association between the pain score in experimental group. }\end{array}$ & Indumathi et al 2018 \\
\hline & $\begin{array}{l}\text { There was a significant difference between the mean pain scores of } \\
\text { mothers in experimental group and control groups }\end{array}$ & Saima et al 2018 \\
\hline
\end{tabular}

Out of which one study done in 2018 by Saima et al on effectiveness of back massage on pain among primigravida mothers during first stage of labour used pre-test and post-test control group design.
Study conducted by Choudhary et al used multiple time series design, study by Gounder et al used post-test only control group design. Studies by Kavitha et al, Devi et al, Zaghloul et al and Indumathi et al used visual analogue 
scale to measure the intensity of labor pain. Devi et al used both pain intensity scale and visual analogue scale to assess the intensity of labor pain. Another studies reported by Choudhary et al, Sriasih et al, Khavandizadeh et al, Panchal et al, Chauhan et al used numerical pain rating scale to assess the pain in pregnant women during labour. Only one researcher Saima et al used Wonback's pain assessment scale to assess the pain among primigravida mothers during first stage of labour. Studies by Choudhary et al and Sriasih et al used non-probability consecutive sampling technique. Studies by Devi et al and Saima et al used random sampling technique. Studies by Kavitha et al, Khavandizadeh et al, Zaghloul et al, Sheoran et al, Panchal et al and Chauhan et al used purposive sampling technique. For analysis of data out of 10 studies 3 studies Sriasih et al, Khavandizadeh et al and Zaghloul et al used chi-square and Mann-Whitney tests. Studies by (Sheoran, et al and Devi et al used ANOVA and $\mathrm{t}$ test. One study by Choudhary et al used ANOVA for analysis. All other studies like Kavitha et al, Khavandizadeh et al, Zaghloul et al, Sheoran et al, Devi et al, Malliga et al, Chauhan et al, Indumathi et al and Saima et al used t test. All studies except one study by Sheoran et al included for review of literature reported a significant difference at the level of $p<0.05$ between the experimental and control group on labour pain and There was significant association between the pain score in experimental group. This reveals the effectiveness of massage in the intensity of labour pain in pregnant women. Characteristics of all papers included for this study is mentioned in (Table1).

\section{DISCUSSION}

Although the similarities across the studies; there were some differences in sample size and research design but in all studies researcher used back massage method to reduce the labour pain and pain perception score checked by numerical pain scale, visual analogue scale and Wonback's pain assessment scale. The study finds that there was significant difference in pain perception score of patients receiving back massage with different types of methods. The statistical data support that there is a difference in pain perception during back massage among experimental group. This method helps to reduce pain during labour.

Management of labour pain is not a great challenge for mothers but also crucial concern in modern medicine. A study done by Czech et al stated that non pharmacological methods such as water birth, transcutaneous electrical nerve stimulation, aromatherapy, acupuncture and acupressure, massage techniques are helpful to reduce labour pain. ${ }^{15}$ A study also suggest that the manual methods like reflexology and massage are effective for management of labour pain, in this study massage helps to manipulating body soft tissue and also helpful to relaxation, so reduction of tension is turn may relieve labour pain same as reflexology also effect the body. ${ }^{16}$ Study done by Thomson et al also demonstrated that Women have mixed experiences of different non-pharmacological and pharmacological pain relief methods. In which women views are varied in their opinion but no matter this study conclude that methods were more effective in relieving pain during labour. ${ }^{17}$

Breathing exercises with (deep inhalation and exhalation) in pregnant women are effective in reducing the labour pain and also shortening the duration of the second stage of delivery. ${ }^{18}$ The methods of non-pharmacological interventions hold promise to relieve pain during labour with no harm or minimal effect to foetus as well as mother also and labour progress are cost effective and simple. ${ }^{19}$

\section{CONCLUSION}

It can be concluded that there are several studies that reviewed and provided a well understanding of interventions that could help to develop more effective intervention to reduce labour pain. All studies conclude effectiveness of back massage on pain reduction during labour in all the mothers such as: primigravida and nulliparous women, based on quantitative data in different country. Current review provides valid evidence for effectiveness of back massage to relieve labour pain. Hence the use of massage therapy can be suggested in the all women during labour pain.

\section{Recommendations}

Further studies of ability and effectiveness are essential to conclude the clinical applicability of back massage in other types of pain. Additional research is needed to imitate the helpful findings of back massage in a more sample.

\section{Funding: No funding sources \\ Conflict of interest: None declared \\ Ethical approval: Not required}

\section{REFERENCES}

1. Sahile E, Yemaneh Y, Alehegn A, Niguse W, Salahuddin M. Practice of Labour pain management methods and associated factors among skilled attendants working at general hospitals in Tigray Region, North Ethiopia: hospital based crosssectional study design. Health Sci J. 2017;11(4):516.

2. Choudhary S, Prakash K, Mahalingam G, Mahala P. Effectiveness of labor support measures on the pain perception of mothers in labor. Int J Med Sci Public Heal. 2018;7(5):1.

3. Hosseni SF, Pilevarzadeh M, Vazirinasab H. Nonpharmacological strategies on pain relief during labor. Biosci Biotechnol Res Asia. 2019;13(2):1-12.

4. Dekker R. Massage for pain relief during labor. Available at: https://evidencebasedbirth.com/ massage-for-pain-relief-during-labor/. Accessed on 25 January 2021. 
5. Pregnant full body massage at home. Available at: https://www.webmd.com/baby/pregnancy-andmassage\#1. Accessed on 25 January 2021.

6. Kavitha P. A study to assess the effectiveness of paced breathing versus back massage for labour pain among antenatal mothers during labour process. Int $\mathrm{J}$ Allied Med Sci Clin Res. 2013;1(2):82-8.

7. Sriasih NGK, Hadi MC, Suindri NN, Surati GA, Mahayati NMD. The effect of massage therapy using frangipani aromatherapy oil to reduce the childbirth pain intensity. Int J Ther Massage Bodyw Res Educ Pract. 2019;12(2):18-24.

8. Aghdam SK, Adib A, Kazemzedeh R. The effects of massage during labor on pain and length of delivery in nulliparous women. Iran J Obstet Gynecol Infertil. 2013;16(83):15-20.

9. Zaghloul MG, Mossad AAM. Effect of effleurage on pain severity and duration of labor among laboring women. IOSR J Nurs Heal Sci. 2018;7(6):1-9.

10. Sheoran P, Panchal R. A study to assess the effectiveness of abdominal effleurage on labor pain intensity and labour outcomes among nullipara mothers during first stage of labor in selected hospitals of district. J caring Sci. 2015;4(1):1585-90.

11. Devi RF, Sangeetha C. Effectiveness of routine back massage versus jasmine oil back massage versus coconut oil back massage on labour pain among parturient mothers in selected tertiary care hospital Bangalore. Int J Health Sci Res. 2016;6:219-24.

12. Chauhan K, Rani S, Bansal P. Effectiveness of olive oil back massage on reduction of labor pain during first stage of labor. Int J Nurs Midwif. 2016;3:2-3.

13. Indumathi A. Effectiveness of ice massage in reduction of labor pain during first stage of labor among primigravida mothers. Emerg Life Sci Res.
2018;4(1):11-4.

14. Saima NJH. A study to assess the effectiveness of back massage on pain among primigravida. Int $\mathbf{J}$ Curr Res. 2018;10(9):73358-60.

15. Czech I, Fuchs P, Fuchs A, Lorek M, TobolskaLorek D, Drosdzol-Cop A, et al. Pharmacological and non-pharmacological methods of labour pain relief establishment of effectiveness and comparison. Int J Environ Res Public Health. 2018;15(12):1-14.

16. Smith CA, Levett KM, Collins CT, Dahlen HG, Ee CC, Suganuma M. Massage, reflexology and other manual methods for pain management in labour. Cochrane Database Syst Rev. 2018;2018(3):1-88.

17. Thomson G, Feeley C, Moran VH, Downe S, Oladapo OT. Women's experiences of pharmacological and non-pharmacological pain relief methods for labour and childbirth: A qualitative systematic review. Reprod Health. 2019;16(1):1-24.

18. Yuksel H, Cayir Y, Kosan Z, Tastan K. Effectiveness of breathing exercises during the second stage of labor on labor pain and duration: a randomized controlled trial. J Integr Med. 2017;15(6):456-61.

19. Boateng EA, Kumi LO, Diji AKA. Nurses and midwives' experiences of using non-pharmacological interventions for labour pain management: A qualitative study in Ghana. BMC Pregnancy Childbirth. 2019;19(1):31088408.

Cite this article as: Choudhary S, Jelly P, Mahala P, Mery A. Effect of back massage on relieving pain during labour: a systemic review. Int J Reprod Contracept Obstet Gynecol 2021;10:2466-72. 